\title{
The Importance of Eliciting a Good History in Patients Suffering from Vertigo
}

\author{
Vicky S Khattar, Bachi T Hathiram
}

\begin{abstract}
Vertigo is a symptom of multifactorial etiology. The presentation of this symptom can range from a single acute episode, to recurrent episodes, and can also change it's nature and frequency; it thus becomes prudent for the clinician to be able to screen these patients into those that are caused due to inner ear pathologies, from those that have a neurological, psychological, metabolic, cardiac or other etiology.

As with any medical symptom, the art of eliciting a detailed and thorough history from the patient can help the clinician to pinpoint the 'culprit' system/organ responsible for this symptom, and the necessary steps can also be taken. It also helps to discern those patients that would require urgent medical assistance, and prioritize the care or consultations that they would be directed to. At times, vertigo may be the only presenting symptom of a stroke, and in such cases, the discretion of the physician may even prove to be life saving!

Described below is a review of the literature highlighting the importance and value attached to taking a 'thorough' history of a patient presenting with vertigo.
\end{abstract}

Keywords: Vertigo, History.

How to cite this article: Khattar VS, Hathiram BT. The Importance of E liciting a Good H istory in Patients S uffering from Vertigo. Int J Otorhinolaryngol Clin 2012;4(1):1-4.

\section{Source of support $\mathrm{Nil}$}

Conflict of interest: None declared

\section{INTRODUCTION}

A s with most things in medicine, a particular diagnosis can almost always be ascertained $80 \%$ of the time by taking an accurate history. ${ }^{1}$ The same holds true for vertigo.

There have been many developments in the field of balance and vestibular testing but, as in other branches of medicine, nothing replaces a good clinical history. In addition, the techniques used and ranges of normality adopted by different vestibular laboratories can be very disparate and so 'positive', 'objective' vestibular findings must be taken strictly within the appropriate clinical context. Reciprocally, some clinical histories are so typical, that the absence of corroborative findings on a single visit to the clinic should not make the clinician rule out a diagnosis. ${ }^{2}$

Bayer et al ${ }^{10}$ (2010) developed diagnostic indices based on self-reporting for four commonly occurring vertiginous disorders namely benign paroxysmal positional vertigo (BPPV), vestibular migranie (VM ), phobic postural vertigo (PPV) and M eniere's disease (M D). These were developed as screening tools, to preselect patients and focus on their main symptoms. They demonstrated that some characteristics in these indices had individually a very low sensitivity and specificity, but the same increased when they were combined with the other characteristics. They have also categorically mentioned that these indices were not to be used as sole diagnostic tools, and could never replace a medical consultation and clinical examination of the patient. This became more important when patients were suffering from multiple causes of vertigo, such as PPV occurring after organic vestibular disorders, or complicated BPPV and so on. Nevertheless, these would serve as good epidemiological tools.

\section{Factors to be Assessed while Eliciting a 'History'}

Enumerated below are a few aspects of the 'history taking' process which will help the clinician in arriving at a provisional diagnosis for a vertiginous patient.

\section{Predisposing Factors}

A detailed account of the predisposing factors can divulge a large amount of information about the etiology and triggering factors for a patient suffering from vertigo. Patients who have had a previous history of trauma (external, surgical or even barotrauma) may be suffering from a perilymph or labyrinthine fistula. Patients with a history of convulsions or headaches may be suffering from some coexistent intracranial pathology; the latter symptom may also be seen in patients with vestibular migraine, wherein the headaches and vertigo may coexist. A past history of an upper respiratory infection may help in diagnosing cases of vestibular neuritis or labyrinthitis.

\section{Onset of Vertigo}

It may not be the rule, but vertigo of 'peripheral' origin usually presents with a sudden onset, as compared to that due to 'central' causes, which presents in a more gradual manner. The exception to the latter is seen in patients suffering from acute cerebellar ataxia due to hemorrhage or infarcts, or sudden transient ischemic attacks, wherein the symptoms are sudden in onset. N umerous authors have shown that a small stroke within the posterior fossa can present as acute severe dizziness, closely mimicking 
vestibular neuritis. ${ }^{4-6}$ In fact, some series have reported that dizziness was typically abrupt in onset in patients with a 'cerebrovascular' cause of acute dizziness. ${ }^{12,13}$

\section{Duration, Chronology and F requency of Symptoms}

The symptoms of BPPV usually last for a few seconds and may even last up to a minute. The same goes for postural hypotension, in which the blood pressure stabilizes within a minute and the symptoms disappear. Given the completely different nature of the two pathologies, it is not difficult to differentiate between the two. The point to remember in BPPV is that the patient is normal in between episodes. Occasionally mild light headedness or nausea can persist longer than a minute, but any patient presenting with giddiness lasting longer than a minute should be investigated for other potential causes. ${ }^{7}$

When the symptoms persist for a few hours, and gradually settle down, reducing in severity over a few days to sometimes weeks, one may think of a probable diagnosis of a unilateral vestibular deficit as seen in cases of vestibular neuritis and labyrinthitis. Occasionally, the rotatory vertigo will cease after a few days, and only a mile imbalance persists; this is classically seen in unilateral vestibular losses.

Fluctuating symptoms are often seen in patients suffering from MD.

The frequency of the symptoms, coupled with their association with the respective triggering factors may sometimes be all that is required to diagnose conditions, such as BPPV, perilymph fistulae, etc.

\section{Aggravating Factors}

This is one of the most important aspects of eliciting the history. Such 'triggers' are a key source of diagnostic information, especially in patients with transient dizziness. ${ }^{11}$ B elow is a table enumerating the various 'triggers' and their associations with vertiginous disorders:

\begin{tabular}{ll}
\hline Triggers & $\begin{array}{l}\text { Association with } \\
\text { vertiginous disorder }\end{array}$ \\
\hline $\begin{array}{l}\text { 1. Body postural changes } \\
\text { (mention which posture) }\end{array}$ & $\begin{array}{l}\text { BPPV, postural } \\
\text { hypotension }\end{array}$ \\
2. Neck position & Cervicogenic vertigo \\
3. Standing & Postural hypotension \\
4. Eyes closed & Bilateral vestibulopathy \\
5. Straining & Perilymph fistulae \\
6. Hyperventilation & Hyperventilation syndrome \\
7. Public places & Visual vertigo \\
(supermarket, crowded & \\
places) &
\end{tabular}

8. Loud noise

9. M oving in dark

10. Mal de debarquement

11. Walking on soft terra firma/wearing soft soled shoes

12. M otion intolerance

13. Visual triggers

Relieving Factors

A s per the respective triggering factors, a list of the relieving factors should also be made, and correlated with the natural progression of a particular disorder.

\section{Comorbidities/Associated Symptoms}

An association with hypo/hyperacusis, tinnitus, aural fullness or fluctuating hearing loss may indicate that the patients is suffering from an otological cause, such as a M eniere's syndrome. However, one must remember that the blood supply to the inner ear and related structures can often be compromised in cerebrovascular episodes, and these too may lead to auditory symptoms. It may be for this reason that patients with auditory symptoms have been excluded from numerous studies of acute vestibular syndrome and vestibular neuritis. ${ }^{14}$ The presence of nausea, vomiting, visual disturbances, blackouts, TIAs, perioral paresthesia, speech or swallowing problems or ataxia may indicate a 'central' cause of the vertigo. Facial weakness may indicate a lesion in the cerebellopontine angle or the internal acoustic meatus. However, mild double vision can also result from a peripheral vestibular lesion, and this is thus not a reliable discriminator. ${ }^{7}$

\section{Proportionality of Associated Symptoms}

U sually peripheral vestibular disorders are characterized by autonomic, gait and postural symptoms that are proportional to the dizziness experienced by the patient, while they are disproportional in patients suffering from central vestibular disorders. ${ }^{14,15}$

\section{Oscillopsia}

Movement of the visual field with head movements is termed as oscillopsia. It is usually a symptom of bilateral 
vestibulopathy, but may also be seen in patients suffering from central nervous system (CNS) disorders, wherein it may be related to only certain head movements, and may sometimes even be unrelated to any head movements.

\section{Medical History}

A thorough medical history is essential to understand the underlying comorbidities that may predispose or aggravate the symptoms, and draw attention to the treating clinician to the possibility of drug interactions, should the patient be prescribed any medications.

\section{Psychological Factors}

These should al so be considered when evaluating a patient with vertigo. Often they are the only reason for persistence of the vertiginous symptoms after the actual disorder has been tackled, such as in phobic postural vertigo. In addition, the type of personality may have an important role in the eventual treatment of the patient.

\section{Drug Intake}

Drugs may have been consumed for a variety of reasons: Some patients may be self-medicating with over-the-counter medications which include a variety of sedatives; others may be treated for a varied period of time by their primary care physicians; yet others may be simply consuming antivertiginous drugs for symptomatic relief by their physicians without any rationale for their prolonged use. Finally, there may be a group of patients who may be under the influence of addictive substances, such as al cohol or marijuana, which may by themselves be the primary cause of the vertigo.

There has also been some mention of avoidance of any medications prior to vestibular testing for a period of 24 to 48 hours; while this may hold true for patients consuming sedatives, it is not so for those taking drugs, such as betahistine (which have no sedative effects). It is important that the patients feel comfortable while being tested and avoidance of their medications for a period of 1 to 2 days may only make the patient miserable.

\section{Family History}

There have been numerous studies that have highlighted the familial occurrence of vertiginous disorders. In a study by $C$ ha et al (2007), ${ }^{3}$ it was seen that the frequent association of episodic vertigo, migraine and MD in closely related individuals, including identical twins supports the heritability of a migraine-M eniere's syndrome.

\section{Common Misconceptions}

One must understand that al though reliance on the patient's history is a vital part of arriving at a diagnosis of the vertiginous disorder, this may not al ways be true. A recent physician survey by Stanton et al $(2007)^{8}$ revealed certain common misconceptions regarding dizziness presentations.

The first being that isolated vertigo/dizziness as the only symptom, reduces the likelihood of the patient suffering from a stroke; while this may be true for a vast majority, it must always be kept in mind that a stroke can very closely mimic vestibular neuritis. ${ }^{4-6}$

A second common misconception was that patients suffering from spinning vertigo were more likely to have a stroke as compared to those that complained of just lightheadedness or other vague dizziness sensations. A population-based study however, showed that the nature of the dizziness may not always correlate with the pathology; in other words, patients suffering from true vertigo did not have a higher incidence of strokes as compared to those complaining of mere imbalance/dizziness/light headedness. ${ }^{9}$

Thirdly, another misconception was that dizziness exacerbated by head motion was usually due to a peripheral disorder, when in fact, ANY dizziness can be exacerbated by head motion. ${ }^{8}$ The authors of this survey have also mentioned the nonreliability of computed tomographic (CT) scans in ruling out strokes, especially in the acute setting, and in cases with posterior fossa lesions. This however, is out of scope of this article. The primary reason for these above mentioned misconceptions is the over-reliance on patient's symptoms and also CT scans. Thus, the clinical examination plays an important role, and coupled with the history can give an accurate diagnosis. Having said that, one cannot take away from history-taking, its importance.

\section{SUMMARY}

It is vital to remember that a good elicitation of the patient's history in his/her own words, coupled with a few leading questions by the clinician can help the latter at arriving at a provisional diagnosis. This does not mean that the patient would not require a detailed evaluation and vestibular testing from time to time, but the provisional diagnosis obtained after taking the patient's history surely helps in targeting the testing, and arriving at a diagnosis, which is seldom different from that hypothesized from the history. O ne must however, be cautious of overly emphasizing the importance of history-taking, and be aware of certain pitfalls with the same. Having said that, history-taking is an integral and irreplaceable part of the vestibular evaluation; it assumes 
increasingly important roles in the emergency department, in triaging patients and making appropriate referrals.

\section{ACKNOWLEDGMENT}

The authors are thankful to the Dean, TN M edical College and BY L N air Charitable Hospital for granting permission to publish this article.

\section{REFERENCES}

1. Weber PC. History and physical examination in vertigo and dysequilibrium: A practical guide to diagnosis and management. Edited by W eber PC. Thieme M edical Publishers Inc. N ew Y ork: USA 2008;1:1-3.

2. Bronstein AM. Evaluation of balance. In Scott-Brown's Otorhinolaryngology, Head and N eck Surgery 7th ed, Edward A rnold (Publishers) Ltd 2008;3:3706-47.

3. Cha Y H, Kane MJ , Baloh RW . Familial clustering of migraine, episodic vertigo, and M énière's disease. Otol Neurotol 2008 Jan;29(1):93.

4. Norrving $B, M$ agnusson $M, H$ oltas $S$. Isolated acute vertigo in the elderly; vestibular or vascular disease? A cta N eurol Scand $1995 \mathrm{~J}$ an;91(1):43-48.

5. Lee H, Cho Y W. A case of isolated nodulus infarction presenting as a vestibular neuritis. J Neurol Sci 2004 J un 15;221(12): 117-19.

6. Lee H, Sohn SI, Cho Y W, et al. Cerebellar infarction presenting isolated vertigo: Frequency and vascular topographical patterns. Neurology 20060 ct 10;67(7):1178-83.

7. Kerber KA . V ertigo and dizziness in the emergency department. Emerg M ed Clin N orth A m 2009 Feb;27(1):39-50.

8. Stanton V A, H sieh Y H, Camargo CA J r, et al. Over-reliance on symptom quality in diagnosing dizziness: Results of a multicenter survey of emergency physicians. $\mathrm{M}$ ayo $\mathrm{Clin}$ Proc 2007 N ov;82(11):1319-28.

9. Kerber KA, Brown DL, L isabeth LD, Smith M A, M orgenstern $L B$. Stroke among patients with dizziness, vertigo, and imbalance in the emergency department: A population-based study. Stroke 2006 Oct;37(10):2484-87.
10. Bayer 0, W arninghoff JC, Straube A. Diagnostic indices for vertiginous diseases. BM C N eurology 2010;10:98.

11. Tarnutzer AA, Berkowitz $A L$, Robinson $K A$, H sieh $Y H$, Newman-Toker DE. Does my dizzy patient have a stroke? A systematic review of bedside diagnosis in acute vestibular syndrome. CM A J, 2011, J une 14;183(9).

12. Gomez CR, Cruz-Flores $S, M$ alkoff M D, et al. Isolated vertigo as a manifestation of vertebrobasilar ischemia. Neurology 1996;47:94-97.

13. Grad A, Baloh RW. V ertigo of vascular origin. Clinical and electronystagmographic features in 84 cases. A rch Neurol 1989;46:281-84.

14. Newman-Toker DE. Diagnosing dizziness in the emergency department- why. "W hat do you mean by 'dizzy'?" should not be the first question you ask [doctoral dissertation, Bloomberg School of Public H ealth]. Baltimore (M D): The J ohns Hopkins University; 2007. A vailable: http://gateway .proquest .com/ openurl ?url_ver $=$ Z39.88-2004\& res_dat =xri:pqdiss\& $\mathrm{rft}$ val fmt =info:ofi/fmt:kev:mtx:dissertation\& rft_dat=xri:pqdiss:3267879 [publication no. AA T 3267879] (accessed 2010 N ov. 19).

15. Edlow JA, New man-Toker DE, Savitz SI. Diagnosis and initial management of cerebellar infarction. Lancet Neurol 2008;7: 951-64.

\section{ABOUT THE AUTHORS}

\section{Vicky S Khattar}

A ssistant Professor, D epartment of ENT and H ead and N eck Surgery Topiwala $\mathrm{N}$ ational M edical Collegeand BY L N air Charitable H ospital M umbai, M aharashtra, India

CorrespondenceAddress: C-7, Swati Kalyan Complex, Panch M arg Off $Y$ ari Road, V ersova, M umbai-4000061, M aharashtra, India Phone: 91-9930977110, 91-9324655600, e-mail: vickykhattar@ rediffmail.com, orlclinics@g mail.com

\section{Bachi T Hathiram}

Professor and H ead, D epartment of ENT and Head and Neck Surgery Topiwala N ational M edical Collegeand BY L N air Charitable H ospital M umbai, M aharashtra, India 